\title{
RCS Modeling and Measurements for Automotive Radar Applications in the W Band
}

\author{
Emna Bel Kamel ${ }^{1}$, Alain Peden ${ }^{1}$, Patrice Pajusco ${ }^{1}$ \\ ${ }^{1}$ Telecom Bretagne MO, Lab-STICC UMR CNRS 6285, Brest, France, emna.belkamel@telecom-bretagne.eu
}

\begin{abstract}
This paper describes a reliable methodology for radar cross section (RCS) measurement of complex small and large targets in the $\mathrm{W}$ band. The backscattering behavior of a small car model was measured in an anechoic chamber along with various automotive related targets in a wide gymnasium. Experimental performance in the anechoic chamber is compared to the simulation results. Our simulation model is based on deterministic scattering centers, determined by high frequency approaches, like the physical optics (PO) and the physical theory of diffraction (PTD). Nevertheless, simulations of realistic large objects are both time consuming and difficult to implement. The proposed measurement configuration enables the extraction of non-predetermined scattering points for large object modeling which will significantly decrease the simulation time for road scenarios in radar applications.
\end{abstract}

Index Terms-millimeter wave, measurement, RCS.

\section{INTRODUCTION}

In the ongoing effort to improve road safety and communication systems between vehicles, robust RCS signatures of automotive targets are crucial. Generating the cartography of a real radar environment is required to design efficient radar systems. Our study is focused on automotive complex radar target modeling and experimental characterization in order to optimize detection systems. The complexity of targets originates from complex shapes as well as various electromagnetic phenomena. This involves simple and multiple reflections, edge and corner diffraction and creeping waves. In the millimeter wave bands, the modeling of electrically large problems by rigorous electromagnetic methods, such as the method of moments (MOM), is a complex task [1]. They are computationally too expensive when the target dimension is several orders of wavelength. The direct use of the asymptotic methods is still applicable but requires weeks to run the simulation. This can be resolved by using the scattering points approach. They are representative of the most important geometric parts in terms of scattering. The complexity of the target can then be reduced to limited number of scattering points. Various techniques for radar target scattering point extraction have been presented in the literature [2]-[4].

A few studies regarding three-dimensional (3D) complex target modeling in terms of simple and multiple reflection and edge diffraction in the $\mathrm{W}$ band can be found [5]-[6]. Indeed, simulation of radar signatures is a challenging task, due to the lack of available high-fidelity electromagnetic models of complex objects such as vehicles. Running these models in the $\mathrm{W}$ band (76 to 81
$\mathrm{GHz}$ for automotive radar) also requires a fairly large memory and computation time, due to the large size of the target in terms of wavelength. Moreover, RCS experiments in an anechoic chamber give accurate results but can be very expensive, depending on the size of the targets. The platform used to hold large objects like vehicles is available in very few research laboratories in the world. Only a limited set of reports on RCS simulation and measurements of vehicles and pedestrians in the $\mathrm{W}$ band have been reported to date [7]-[9]. The main focus of this work is to investigate car backscattering behavior in millimeter wave bands through simulation and measurements.

This paper is organized as follows. Section II describes the RCS model that includes the shadowing and multiplescattering effects. The methodology used to optimize the simulation procedure is also discussed in terms of car model simplification and numerical methods. Section III describes the RCS measurement system associated with an anechoic chamber and a gymnasium. The experimental results are also presented here. Finally conclusions and perspectives are drawn in Section IV.

\section{RCS SIMULATION OF COMPLEX TARGETS}

\section{A. Deterministic scattering center model}

Our Matlab model accounts for shadowing effects by performing a visibility test. The target is discretized into triangular facets to achieve an accurately estimated scattering contribution for each part. Illuminated and shaded surfaces are determined according to the algorithm of Trumbore [10]. A positive result of the scalar product between the normal to the triangle's surface and the direction of the incident wave indicates that a facet is facing the radar. Then, a Z-buffer approach [10] is applied to determine the portions of the target that can be hidden by other parts. Depth information is used to eliminate facets that are not visible.

To compute the RCS, the deterministic approach [2] was used, which requires only modeling target by facets. It is based on a predefined distribution of fixed scatterers. The position of the center of a surface, edge or corner can represent the position of the scattering centers. The amplitude and phase of field originating from a scattering point are computed by asymptotic methods. We then implemented the PO approximation to obtain specular reflection from the target [11]. The scattering points are the centers of the mesh surfaces. The scattered field is computed 
from the radiation integrals of current densities. In the case of a polygonal planar surface, this integral is solved in a relatively simple way as suggested in [12] by transforming the surface integral into a linear integral. Away from the specular directions, the edge effects contribute significantly to the scattered field. The PTD was then developed to overcome the PO limitation, as it takes into account the diffraction by edges. The scattering points are the centers of the edges. The source of the diffracted field is attributed to the fictitious electric and magnetic equivalent currents, along the edge. The expressions given by Michaeli [13], based on the fringe currents existing on the edge, are used. These currents are calculated assuming the straight edge locally around each point of the contour being considered.

\section{B. Simulated car model generation}

The short wavelength in the $\mathrm{W}$ band requires very fine meshes to model a target, hence posing extensive computational overhead (memory and time). At high frequencies, the RCS predictions from coarse mesh become inaccurate. Coarse mesh will not reflect all the details of the target. Indeed, the degree of error is greatly geometrydependent. The facetization representation of the model and the electromagnetic simulation approach are two key aspects of RCS computation accuracy. The vehicle model used in our RCS simulator describes essentially its exterior metallic structure. It is represented by flat triangular facets. The model usually needs to be corrected for overlapped meshes before running the EM simulation. We chose to apply a surface mesh simplification to the car model in order to reduce its complexity and storage requirements, while perceiving the overall shape, volume and boundaries as much as possible. Curved features are modeled well with small flat triangular facets. Thus, discretization error can be minimized to a large degree. Tests demonstrate that the total number of triangular meshes can be reduced by a factor of 2 with MeshLab software.

\section{Simulation results}

In order to describe the performance of our model in terms of CPU time and memory, we first simulated a generic model of a small, 'car-like' object $\left(6.6 \times 3.6 \times 4.3 \mathrm{~cm}^{3}\right)$, shown in Fig. 1 (a). The results were further compared with the Multilevel Fast Multipole Method (MLFMM) in FEKO, considering it as a reference method. The size of the model was selected due to the memory limitations when simulating a car having a realistic size at $77 \mathrm{GHz}$ with MLFMM in FEKO. 203044 mesh triangles are needed to simulate the mini car model with MLFMM, and only 30 triangles with our Matlab code. This coarse mesh is justified by the $\lambda / 16$ criterion [14]. The RCS simulated by our model is in accordance with the MLFMM, as it will be shown in section III. It also reduces requirements in terms of computational resources. The time resource varies from 384 seconds for our model compared to approximately 4-5 days with the MLFMM. The model is then applied to a simplified pseudorealistic car model (Fig. 1 (b)), and compared to FEKO using the large physical optic (LE-PO) solution. The MLFMM method cannot be applied to this car model as it is electrically very large. An excellent agreement can be seen in Fig. 2 between the results obtained by each of PO, PTD and LE-PO solutions. Nevertheless, the deterministic scattering center model developed in Matlab is not yet optimized. It took approximately five days using 7962 meshes, due to the large number of deterministic scattering points required. Thus, the RCS model should be enhanced by RCS measurements of real automotive targets. High frequency asymptotic methods can be applied to scattering centers extracted from experiments, instead of deterministic ones. The number of scattering points is reduced, as is the computation time.
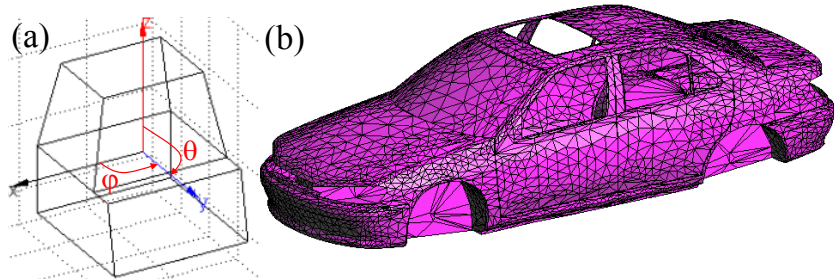

Fig. 1. (a) Car_like model, (b) Sedan car model meshed with 7962 facets

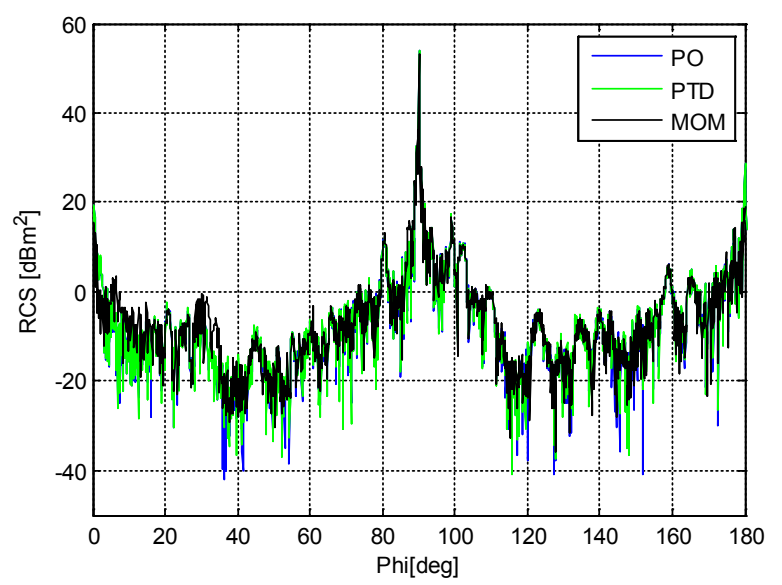

Fig. 2. RCS of the sedan car of Fig. 1 for $\theta=90^{\circ}$

\section{MEASUREMENT SETUP AND RESULTS}

\section{A. Anechoic chamber measurements}

In order to validate the RCS model, an automated characterization bench for RCS was developed. It is composed of a vector network analyzer (VNA) from Rohde \& Schwarz (ZVT20), installed outside the anechoic chamber. It is attached to millimeter wave heads to extend the measurement to the $72-110 \mathrm{GHz}$ band. Due to the difficulty involved in placing the VNA and the millimeter heads in the anechoic chamber $\left(3 \times 1.12 \times 1 \mathrm{~m}^{3}\right)$, they were installed outside it with its door kept open during measurements. A quasi monostatic configuration was used with two horn antennas, operating in the $72-110 \mathrm{GHz}$ range. The measured horn antenna gain is about $23.5 \mathrm{dBi}$. The azimuth and elevation beamwidth angles are about $26^{\circ}$ and $22^{\circ}$ 
respectively, which is sufficient to ensure a uniform illumination of the target, as shown in Fig. 3. The target was placed inside the anechoic chamber. It was mounted on a foam stand placed on a stepper motor at a $2.6 \mathrm{~m}$ distance from the antennas, ensuring the farfield condition. The 38 $\mathrm{GHz}$ bandwidth for the RCS measurement band provided a high range resolution of $4 \mathrm{~mm}$. The whole bandwidth was swept across 2048 points to consider both the target response and echoes in the time domain. It gives an unambiguous range of about $61 \mathrm{~m}$. Measurements were fully automated by remote control of the rotation of the stand and the VNA. The complex $\mathrm{S}_{21}$ parameter was recorded from the VNA over the entire bandwidth, at each angle of given measurement scenario.

It is worth mentioning that the stand foam should be taken into consideration, because its scattering contribution becomes important compared to the low target scattering at millimeter bands, and can potentially influence the measurement results. Therefore, an extra tilted absorber was placed in front of the foam stand and its position and angle were optimized during a series of preliminary tests in order to minimize the stand reflections.

The measurements were carried out in three steps. The backscattered signal from the target was first measured. Secondly, a reference target measurement with known RCS was performed to eliminate dependence on the measurement system. The calibration object chosen here was a $6 \mathrm{~cm}$ diameter sphere since its RCS can be determined analytically and it does not require angular alignment. It was followed by an empty room measurement, made under exactly similar conditions as the RCS measurements but without any target present on the stand. All frequency-dependent measurements were transformed in the time domain by an inverse Fourier transform. These impulse responses were subtracted from an empty room measurement to reduce unwanted echoes. A time gating centered on the object response followed by an FFT refined the frequency domain response and thus the RCS. The resulting signal was then divided by the $6 \mathrm{~cm}$ metallic sphere. This indirect calibration [15] was chosen for all the measurements presented here because of the improved accuracy compared to direct calibration. As only a vertical polarization was used in all measurements, a single reference target at this polarization was needed. Nevertheless, measurements using horizontal polarization or crosspolarization configuration can be performed with the test bench.

After validating the RCS measurement setup, a campaign of angle and frequency RCS measurements was carried out on a series of canonical and complex objects. Figure 4 gives the comparison of RCS distribution measurement results from $-180^{\circ}$ to $180^{\circ}$ of a car-like object in the $72-110 \mathrm{GHz}$ frequency band. This object is made from ROHACELL foam and painted with liquid silver. Despite its relatively simple geometry, it includes several scattering mechanisms, especially edge and corner diffractions. Measurement results matched well with simulations obtained by our PTD Matlab code and by MLFMM simulated using FEKO. The deviation at $-90^{\circ}$ between simulation and measurement occurs from tolerances between the simulated model and the real measured one. These experiments validate the setup and calibration procedure.

(a)

(b)

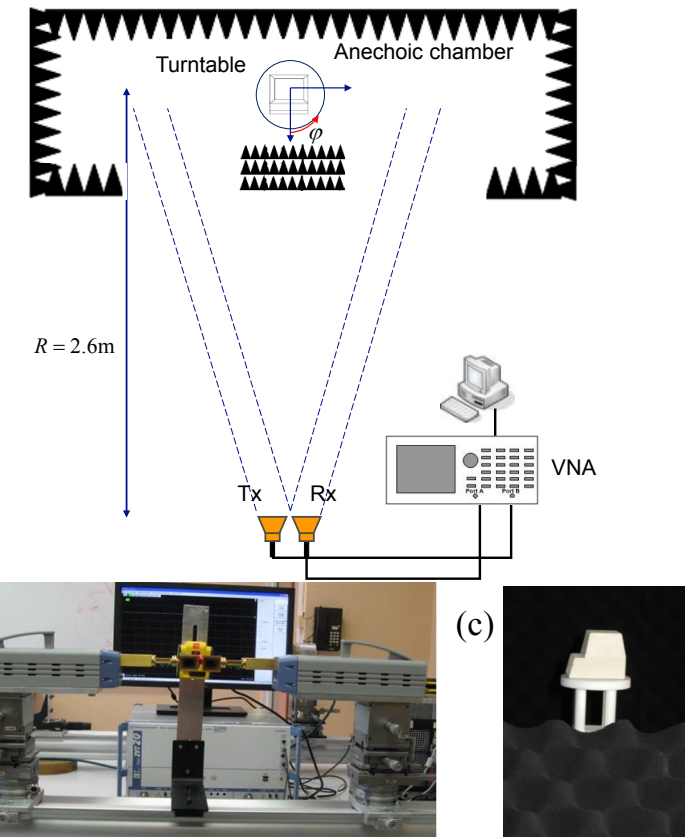

Fig. 3. (a) Millimeter wave measurement configuration, (b) VNA converters in the W-band with rectangular horns, (c) car-like object in foam material (size : $6.6 \times 3.6 \times 4.3 \mathrm{~cm} 3$ )

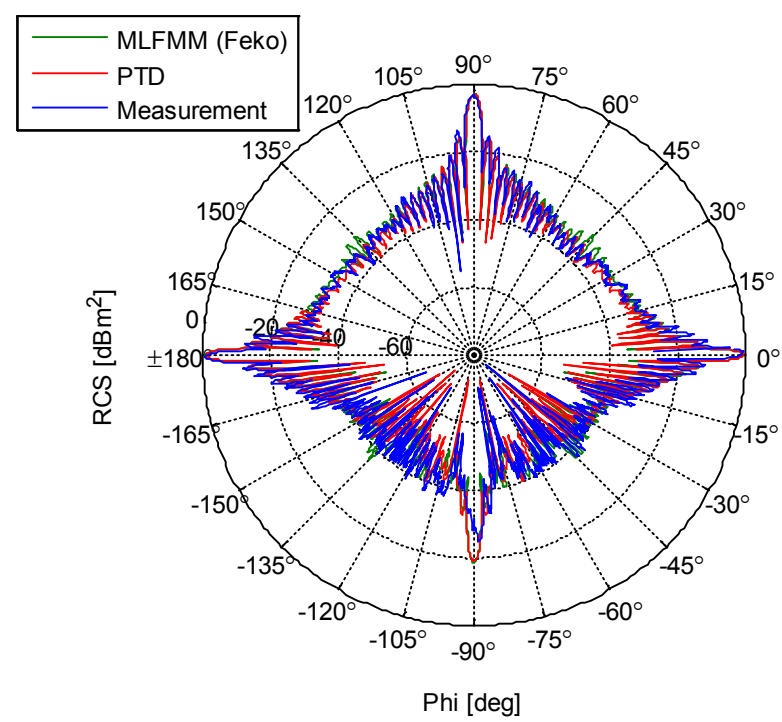

Fig. 4. RCS of car_like for $\theta=90^{\circ}$

\section{B. Measurements in gymnasium}

To characterize the RCS of large objects such as cars, indoor measurements were taken in a large gymnasium. The measurement setup used for the radar cross section measurement is described in section A. The target was placed in the center of the gymnasium, at a distance of $12 \mathrm{~m}$ from the horn antennas. Measurement techniques for large 
targets generally require measuring the RCS in azimuth by rotating the target on a turntable. In our measurement methodology, shown in Fig. 5, a turntable was not needed. Thus, the radar measurement system was driven manually along a circle of $12 \mathrm{~m}$ radius, while the target was fixed. The circle was discretised from $\varphi=0^{\circ}$ to $180^{\circ}$ (assuming the symmetry of the target) with a $2^{\circ}$ angular step. The RCS data was taken at each aspect angle and over the $76-81 \mathrm{GHz}$ bandwidth. No radar-absorbing material was placed around the car during the measurements. Therefore, the resulting backscattered signal contained the target response as well as unwanted contributions from ground and gym components. Direct calibration using the radar equation was applied here due to its simplicity (no reference target is required). The time gating is also of utmost importance to reduce multipath components. The characterization of various cars such as a sedan (Peugeot 406 and Giulietta), a small sedan (Peugeot 107), a van (Renault Trafic), a small van (Peugeot 5008), and other targets (bicycle and pedestrian), was performed. All the measurements were conducted in VV configuration.

a)

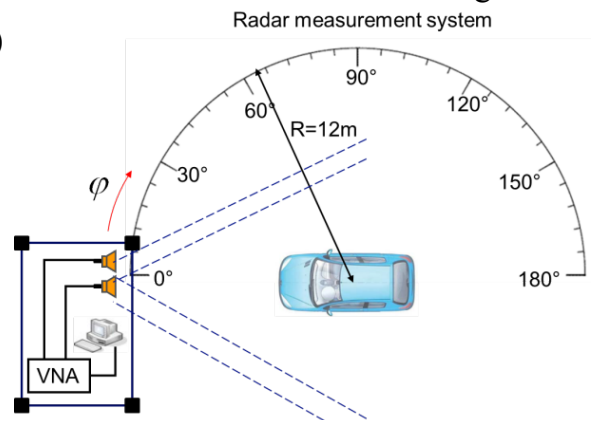

b)

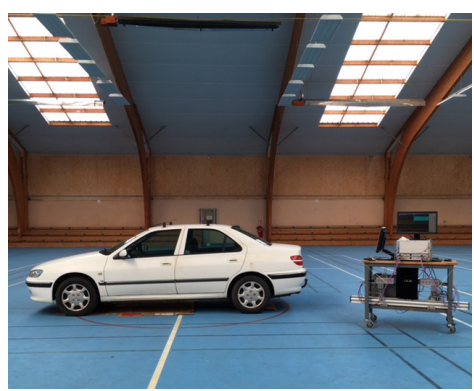

Fig. 5. a) Millimeter-wave measurement configuration , b) measurement environment of the gymnasium

The impulse response deduced from the large band measurement contains maxima in the car range, corresponding to its scattering points. The monostatic RCS of the car is the sum of the contribution from all these scattering centers. Fig. 6 represents the RCS of the cited vehicles. We note a similar behavior of the RCS pattern for all the measured cars. The RCS of the flat part $\left(\varphi=0,90,180^{\circ}\right)$ is significantly larger than that of the corner part $\left(\varphi=45,135^{\circ}\right)$. Moreover, the RCS maximum values vary between $8.7-20.5 \mathrm{dBm}^{2}$ for the front of the cars, $14.4-24.6 \mathrm{dBm}^{2}$ for the back and $19-22 \mathrm{dBm}^{2}$ for the side. These values are in accordance with the results presented in
[7]-[8]. We note that the simulated RCS maximum value for a typical vehicle, presented in Fig. 2, is higher than the measured one, as the wheels are not taken into account in the simulation. Figure 7 shows the RCS of a bicycle and a pedestrian. As expected, both RCS values are smaller than those of cars because there are few or no flat or corner metallic parts. The RCS maximum value of the bicycle is 10 $\mathrm{dBm}^{2}$ in the side view but very weak in the front and back view. The RCS mean value of a pedestrian is around -3 $\mathrm{dBm}^{2}$. In particular, the pedestrian scattering is similar to a cylinder which has an almost fixed RCS pattern for azimuth aspect angles.

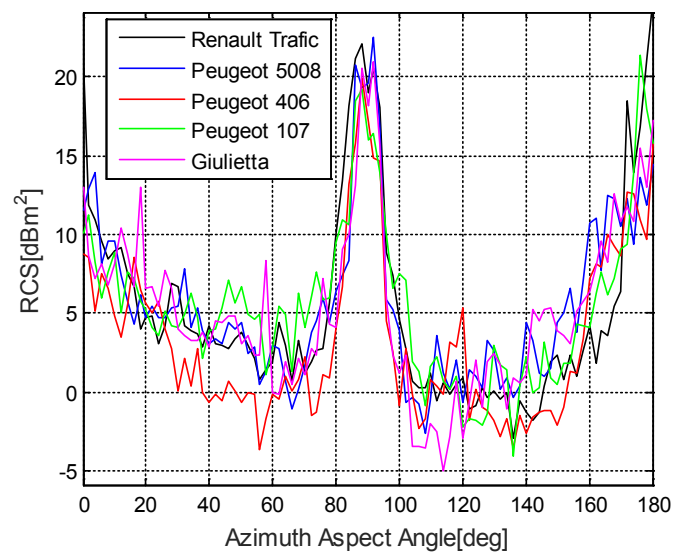

Fig. 6. RCS of five respresentative cars, front view $\varphi=0^{\circ}$

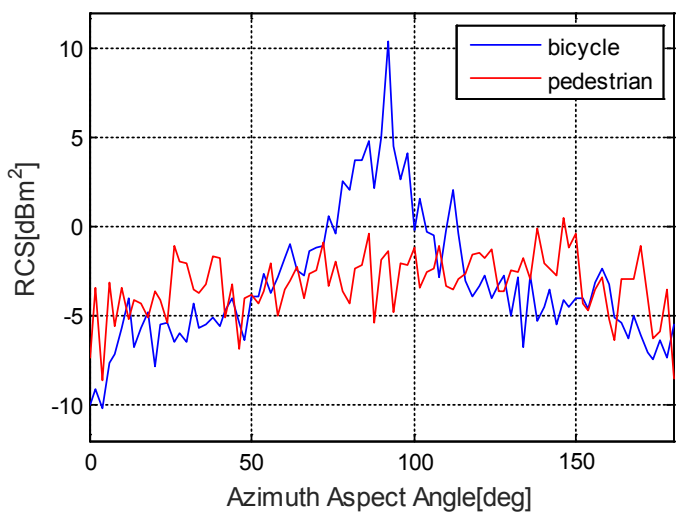

Fig. 7. RCS of a bicycle and a pedestrian, front view $\varphi=0^{\circ}$

Range-azimuth and radar image plots can help locate scattering centers. Based on Fig. 8, three different essential scattering zones (green circles) in azimuth can be identified, at $\varphi=0,90,180^{\circ}$, corresponding respectively to the front, side and back of the car. For the bicycle and pedestrian, there is a roughly uniform distribution of scattering centers. A simple algorithm based on a scattering point approach was implemented to get radar images presented in Fig. 9. The obtained images give a half-top view of the target, highlighting the major scattering points that can be used to reconstruct its RCS. 

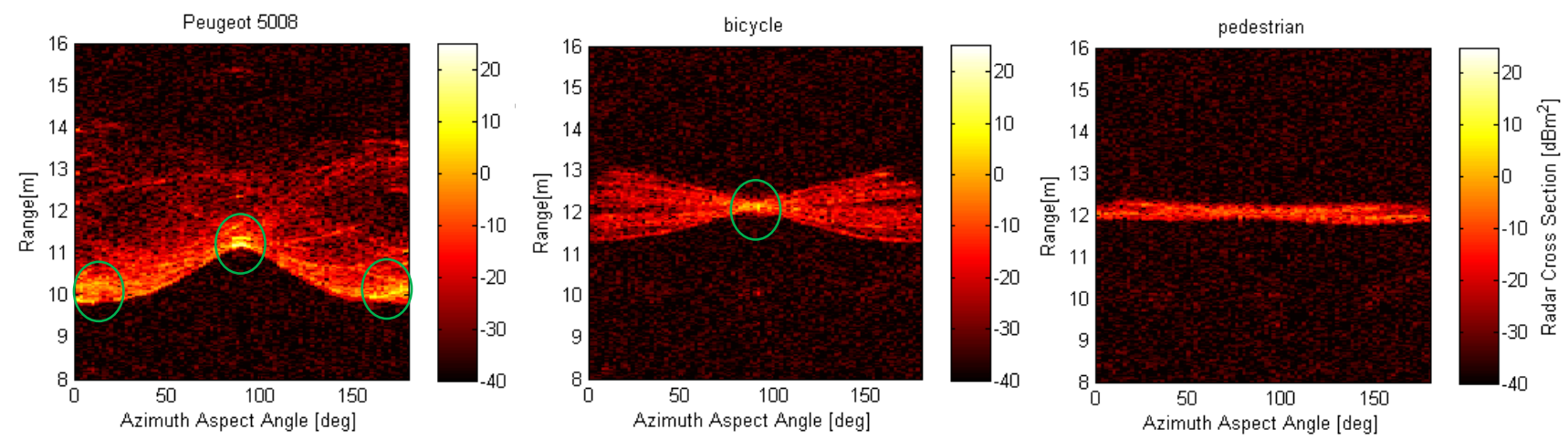

Fig. 8. Range-azimuth plots for three representative targets (car, bicycle, pedestrian)
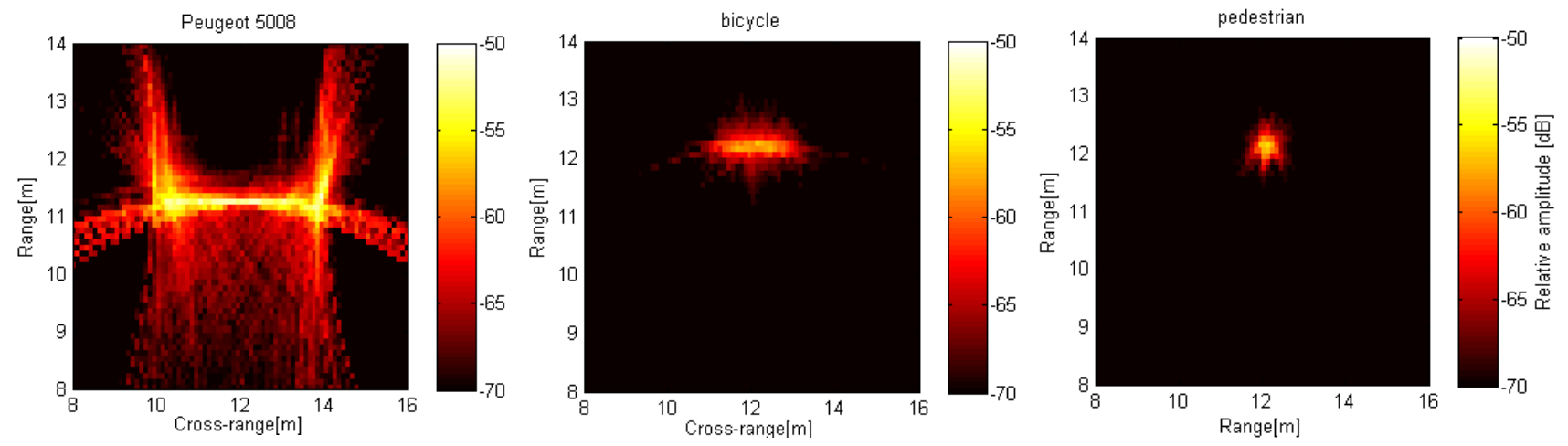

Fig. 9. Radar image plots for three representative targets (car, bicycle, pedestrian)

\section{CONCLUSION}

In this work, a first-order approximation of the RCS of a car in the $\mathrm{W}$ band has been presented by using highfrequency asymptotic methods, while reducing complexity. Accurate measurement results have been obtained for various automotive targets using a cost-effective measurement setup. It was found that the maximum value of RCS for the measured vehicles varies between 19 and 25 $\mathrm{dBm}^{2}$. The maximum value of RCS for the bicycle was found to be $10 \mathrm{dBm}^{2}$, compared to the mean value around -3 $\mathrm{dBm}^{2}$ for a pedestrian. For future perspective, the use of scattering centers extracted by measurement can drastically reduce target model complexity. This can significantly reduce the simulation time for road scenarios in radar applications.

\section{REFERENCES}

[1] R. F. Harrington, 'The Method of Moments in Electromagnetics', $J$. Electromagn. Waves Appl., Apr. 2012.

[2] S.-Y. Wang and S.-K. Jeng, 'A deterministic method for generating a scattering-center model to reconstruct the RCS pattern of complex radar targets', IEEE Trans. Electromagn. Compat., vol. 39, no. 4, pp. 315-323, Nov. 1997.

[3] R. Bhalla, H. Ling, J. Moore, D. J. Andersh, S. W. Lee, and J. Hughes, '3D scattering center representation of complex targets using the shooting and bouncing ray technique: a review', IEEE Antennas Propag. Mag., vol. 40, no. 5, pp. 30-39, Oct. 1998.

[4] K. Schuler, D. Becker, and W. Wiesbeck, 'Extraction of Virtual Scattering Centers of Vehicles by Ray-Tracing Simulations', IEEE Trans. Antennas Propag., vol. 56, no. 11, pp. 3543-3551, Nov. 2008.
[5] M. Chen, M. Kuloglu, and C.-C. Chen, 'Numerical study of pedestrian RCS at 76-77 GHz', in Antennas and Propagation Society International Symposium (APSURSI), 2013 IEEE, 2013, pp. 19821983.

[6] M. Treml, R. Feger, C. Wagner, A. Stelzer, and H. Jäger, 'Radar cross section prediction and measurement at $77 \mathrm{GHz}$ ', EuCAP 2009, 2009.

[7] K. Geary, J. S. Colburn, A. Bekaryan, S. Zeng, B. Litkouhi, and M. Murad, 'Automotive radar target characterization from 22 to $29 \mathrm{GHz}$ and 76 to $81 \mathrm{GHz}$ ', IEEE Natl. Radar Conf. - Proc., pp. 1-6, Apr. 2013.

[8] I. Matsunami, R. Nakamura, and A. Kajiwara, 'RCS measurements for vehicles and pedestrian at 26 and $79 \mathrm{GHz}^{\prime}$, in 2012 6th International Conference on Signal Processing and Communication Systems (ICSPCS), 2012, pp. 1-4.

[9] M. Andres, P. Feil, W. Menzel, H. L. Bloecher, and J. Dickmann, ' 3 D detection of automobile scattering centers using UWB radar sensors at 24/77 GHz', IEEE Aerosp. Electron. Syst. Mag., vol. 28, no. 3, pp. 20-25, Mar. 2013.

[10] N. A. Albayrak, 'RCS computations with PO/PTD for conducting and impedance objects modeled as large flat plates', Thesis, Bilkent University, 2005.

[11] F. Eugene Knott, F. John Shaeffer, and T. Michael Tuley, Radar Cross Section, 2nd edition. Scitech Publishing, Inc., USA, 2004.

[12] W. B. Gordon, 'Far-field approximations to the Kirchoff-Helmholtz representations of scattered fields', IEEE Trans. Antennas Propag., vol. 23, no. 4, pp. 590-592, Jul. 1975.

[13] A. Michaeli, 'Equivalent edge currents for arbitrary aspects of observation', IEEE Trans. Antennas Propag., vol. 32, no. 3, pp. 252258, Mar. 1984.

[14] T. Song and L. Qi-zhong, 'Facet noise in RCS prediction', in 2003 6th International SYmposium on Antennas, Propagation and EM Theory, 2003. Proceedings, 2003, pp. 433-436.

[15] W. Wiesbeck and D. KÄhny, 'Single reference, three target calibration and error correction for monostatic, polarimetric free space measurements', Proc. IEEE, vol. 79, no. 10, pp. 1551-1558, 1991. 